UCRL-LR-103683

Distribution Category UC-401

UCRL-LR- -103683

DE91 001078

\title{
The Unusual Stability of TATB: A Review of the Scientific Literature
}

\author{
Steven F. Rice \\ Randall L. Simpson \\ Energetic Materials Section \\ Department of Chemistry \& Materials Science \\ Lawrence Livermore National Laboratory
}

Manuscript date: July 4, 1990

\section{LAWRENCE LIVERMORE NATIONAL LABORATORY University of California - Livermore, California • 94551}

Available to DOE and DOE contractors from the Office of Scientific and Technical Information

P.O. Box 62, Oak Ridga. TN 37831 Prices available from (615) 576-8601, FTS 626-8401 


\section{Table of Contents}

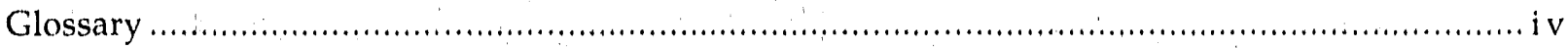

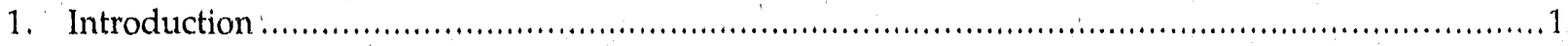

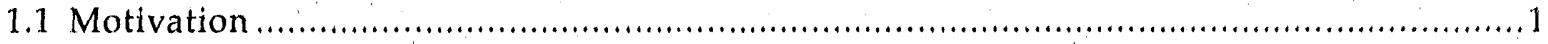

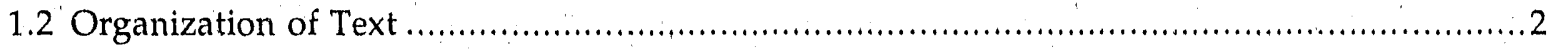

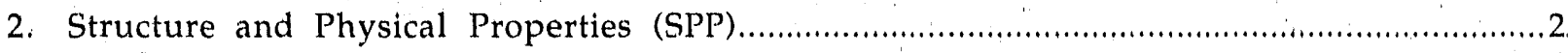

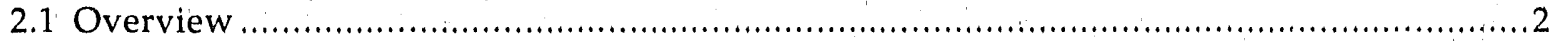

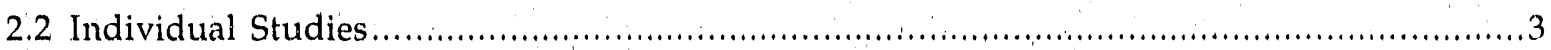

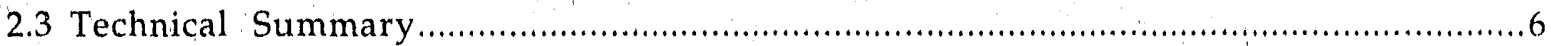

3. Differential Thermal Analysis and Unconfined Decomposition (DTA) ..................................6

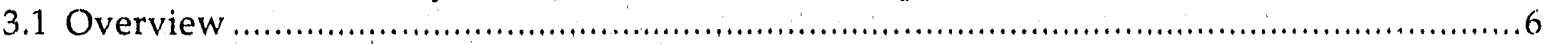

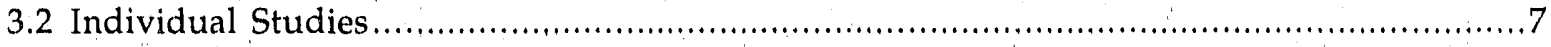

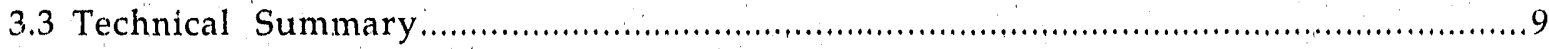

4. Time to Explosion and Confined Pyrolysis (TTX) ....................................................... 10

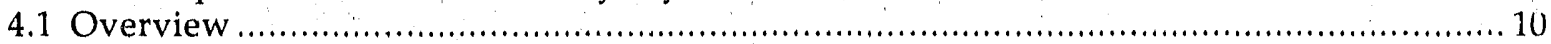

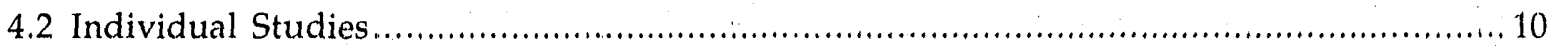

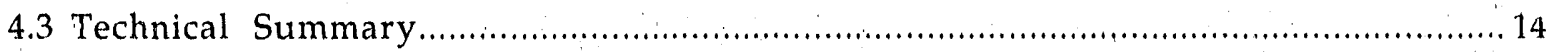

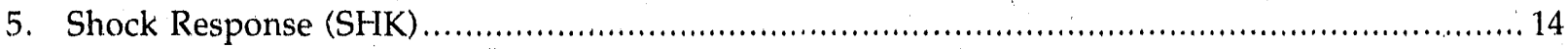

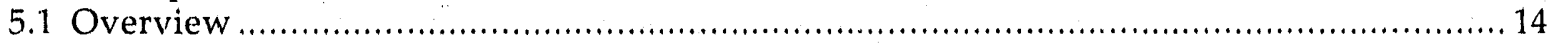

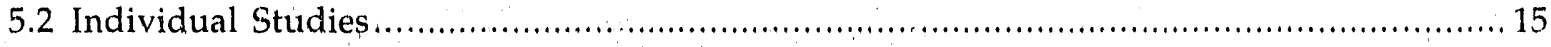

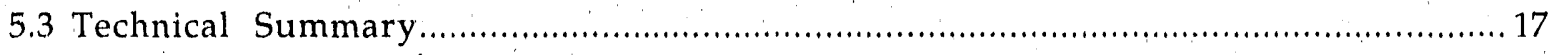

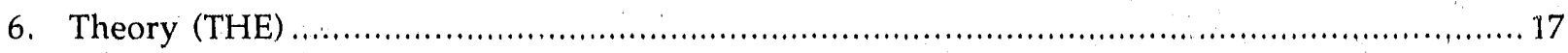

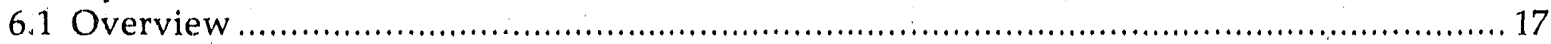

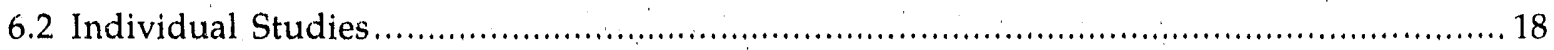

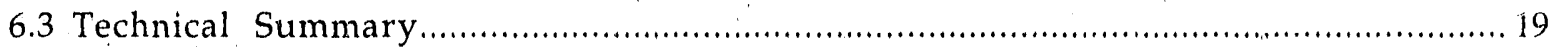

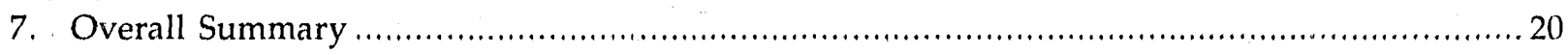

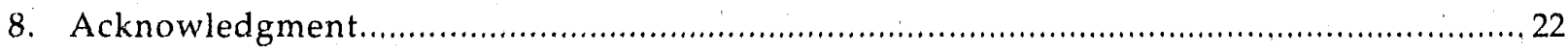

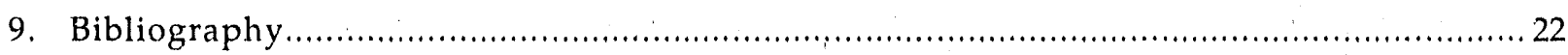




\section{Glossary}

DATB diaminotrinitrobenzene

DSC differential scanning calorimetry

DTA differential thermal analysis

ESR : electron spin resonance

GAPS gross atomic populations

LANL Los Aliamos National Laboratory

LLNL Lawrence Livermore National Laboratory

MATB monoaminotrinitrobenzene, or picramide

NSWC Naval Surface Weapons Center

ODTX one-dimensional time to explosion

SCF

SEM scanning electron microscopy

TATB triaminotrinitrobenzene

TD

$T_{\max }$

TNB

TNT

TOPS

TTX

value obtained from DTA experimental exotherm

critical temperature (temperature at which time to explosion is infinite)

trinitrobenzene

trinitrotcisene

total overlap populations

time to explosion

XPS

$x$-ray photoelectron spectroscopy 


\title{
The Unusual Stability of TATB: A Review of the Scientific Literature
}

\author{
1. Introduction
}

\subsection{Motivation}

The thermal stability of a high explosive (HE) is one of the most important properties affecting its practicality as a constituent of either conventional or nuclear weapons. Therrnal stability is of concern, not only with regard to the formulation, processing, and handling of an HE but also with respect to its safety in a variety of accident scenarios that includes fuel fires, propellant fires, and even more complicated events with a potential for sympathetic detonation. An increased emphasis on such hazards has renewed interest in understanding the fundamental relationship between molecular structure and reactivity of energetic materials.

For quite some time it has been known that 1,3,5-triamino-2,4,6-trinitrobenzene (TATB) is a reasonably powerful high explosive whose thermal and shock stability is considerably greater than that of any other known material of comparable energy. Consequently, it is a very attractive candidate for the main HE charge in a nuclear weapon. The apparent uniqueness of TATB's combination of desirable properties has made it the subject of numerous studies focusing on its unusual stability. Among the more important questions these studies address are: (1) Is TATB the only organic molecule with such high energy and low sensitivity? (2) What are the chemical and structural properties of this molecule that make it so insensitive? (3) How can we use our understanding of the relationship between structure and sensitivity to guide synthesis chemists toward perhaps even better energetic molecular systems?

This, review is designed to fulfill the needs of both the general HE community and the Weapons Program within Lawrence Livermore $\Gamma$ 'ational Laboratory (LLNL). It is intended as an up-to-date review of the scientific literature on TATB since its discovery as a high explosive. In particular, it focuses on clarifying our current understanding of the relationship between the structure of TATB and its unique thermal stability.

We review here a large number of different publications by many authors. In general, the results of the reported experiments agree with one another. However, there is less overlap between experiments than is desirable. And where there are disagreements, it seems that not much effort has gone into reconciling them. Where observations do conflict, we have attempted to explain them. Occasionally, the scientific and theoretical interpretations of some of experiments do not seem warranted by data. In such cases we have attempted to illuminate the source of concern.

Much of the work on TATB appears in papers whose primary focus is on more widely used HEs such as HMX, RDX, and TNT. This review does not attempt to present or interpret the results for these other explosives except where direct comparison with the properties of TATB is of particular significance.

A small portion of the work on "TATB" presented here actually consists of experimental studies on TATB formulated as PBX-9502 or as LX-17. Where relevant, this distinction is indicated. However, inasmuch as this review focuses on thermal response and the relationship of chemical reactivity to the molecular and lattice structure of TATB as a pure material, results from these other formulations may not be directly applicable, and in general we have omitted them. Of specific concern, however, are several studies indicating that many explosives thermally decompose by way of an autocatalytic mechanism, especially in confined environments. Decomposition of a binder simultaneossly with an energetic material could produce molecular fragments that either enhance the overall rate of decomposition or accelerate a rapid transition to explosion. In the case of TATB, the overall purity of a material has been shown to be important in its thermal explosion behavior. Formulated explosives contain some degree of impurity (albeit small) introduced during the formulation process. In many cases 
it is difficult to determine the net effect of this contamination on a variety of explosive properties, thermal response not excepted.

\subsection{Organization of Text}

In this review we summarize the salient results of individual papers. Often, simply reproducing the abstract would represent the reported scientific results fairly accurately. However, because quite a few papers focus mainly on HEs of less interest to this review, our summaries emphasize only the TATB results. In many cases. we offer an opinion about the data in a paper that may not completely agree with all the interpretive statements presented by the authors. We have made every effort to identify clearly these editorial comments.

Summaries of individual papers are grouped together into topic classes. Within each of these classes, summaries are presented chronologically. Each section devoted to a topic class is organized similarly: the more important results of the papers in a class are summarized, together with a brief history. The summary of each paper is presented primarily as a free-standing work. Free-standing summaries of individual papers are followed by a discussion of interpretations of the papers as a group. That is, we attempt to place the papers in the context of a comprehensive description of the current status of the work.

Unfortunately, a number of papers fit into more than one class. When this is the case, we present. the detailed results of a paper in a single topic class but also discuss its germane aspects in the summary of the related class. The detailed results presented in each topic class are followed by a final summary.

Our topic classes are:

- Structure and Physical Properties.

- Thermal Analysis and Unconfined Decomposition.

- Time to Explosion.

- Shock Response.

- Theory.

The first topic class, Structure and Physical Properties, contains papers that report physical data related to molecular or crystal structure, solvent : iclusion, sublimation characteristics, and spectroscopic properties. The Thermal Analysis lass encompasses a variety of studies based on heating an unconfined sample at a known rate and then neasuring a calometric response as a function of time. This section also includes several experiments that are unrelated to thermal analysis but that do examine properties of thermal decomposition in unconfined environments. The Time to Explosion class features one-dimensional time-to-explosion (ODTX) results as well as critical temperature experiments and analysis. If focuses on properties of samples that are heated in pressure-confining conditions. The Shock Response class contains an assortment, but certainly not all, of the important papers on shock initiation of TATB. To maintain our focus, we single out papers that interpret shock or projectile initiation of TATB in relation to the material's chemical structure. Finally, the Theory topic class comprises papers presenting ab initio calculations of TATB's electronic structure and details of its structure in relation to reactivity.

\section{Structure and Physicai Properties (SPP)}

\subsection{Overview}

The compound TATB has been known for its unusual thermal properties since the 1950s. It is a yellow crystalline solid that is insoluble in most solvents. The best solvent is hot, concentrated sulfuric acid. The first important work on the reason for this unique stability was done in 1965 by Cady and Lar on, who determined the crystal structure of TATB. Their paper is the first to identify the presence 
of a strong intra" and intermolecular, hydrogen-bonded network, now presumed by many to stabilize the overall molecular lattice. Theirs is probably the most important work done in attempting to understand this molecule and its properties. Most explanations of other physical measurements performed on this molecular system cite the results of Cady and Larson as at least a partial explanation of newer observations.

Other workers have studied physical properties near the nitrogen atoms in TATB, recording and analyzing complete infrared and Raman spectra as well as x-ray photoelectron and Auger spectra. These studies attempt to relate such measurements to the unusual structure of TATB's molecule or crystal lattice. Also cataloged are TATB's macroscopic properties such as heats of formation and sublimation, compressibility, coefficient of thermal expansion, and thermal diffusivity. Much of this information can be found in B. M. Dobratz, LLNL Explosives Handbook: Properties of Chemical Explosives and Explosive Simulants, Lawrence Livermore National Laboratory, UCRL-52997 (March 16, 1981) and in T. R. Gibbs and A. Popolato, LASL Explosives Properties Data (University of California Press, Burkeley, 1980). The work summarized below emphasizes studies that have attempted to relate experimental measurements to TATB's special thermal properties.

\subsection{Individual Studies}

SPP:1. The Crystal Structure of 1,3,5-triamino,-2,4,6-trinitrobenzene. H. Cady and A. C. Larson, Acta Cryst. 18, 485-496 (1965).

The authors present a detailed crystallographic analysis of 928 x-ray reflections obtained from a single crystal of TATB of dimensions less than $200 \mu \mathrm{m}$. The crystal was grown from a solution of hot nitrobenzene. The structure was refined to an $R$ value of 0.053 in the triclinic crystal class (space group $P \overline{1})$. There are two molecules per unit cell $(Z=2)$ related to each other by the inversion symmetry of the lattice. Only one polymorph was observed. With effort the hydrogen atoms were located, although there are some discrepancies with respect to the thermal parameters. The authors suggest that additional refinement could prove rewarding. They present a detailed sketch of the whole TATB molecule, including bond lengths and angles. The discussion is brief, but several points are made: (1) TATB molecules are arranged in planar sheets parallel to the crystallographic $a-b$ plane. (2) Bondorder calculations suggest that the resonance structure involving an aromatic benzene ring may not be very significant in the true electronic structure of the molecule; the ring is greatly distorted from sixfold symmetry. (3) There is very clear evidence for intramolecular hydrogen bonding involving aimost a complete transfer of a hydrogen from an amino group to one of the adjacent nitro groups. (4) There is some evidence for intermolecular hydrogen bonding, but only within the molecular sheets mentioned above. The normal resonance form (with an aromatic $C_{6}$ ring) is a much less accurate description because of the nature and location of the nitro and amino substituents. A resonance form that is described by a $\mathrm{C}-\mathrm{NH}_{2}$ double bond is enhanced by the strong electron-withdrawing $-\mathrm{NO}_{2}$ group at the ortho and para positions. The crystal structure and bond order calculations clearly indicate the importance of this distorted structure. Ab initio calculations performed much later support this conclusion (see THE:4,5).

SPP:2. Low-Frequency Neutron Spectrum of 1,3,5-Triamino-2,4,6-Trinitrobenzene. V. D. Gupta and B. L. Deopura, Mol. Phys. 19, 589-592 (1970).

The authors report the inelastic neutron-scattering spectrum of TATB from 30 to $900 \mathrm{~cm}^{-1}$. The resolution of the data is not very good, but because the neutron spectrum is particularly sensitive to modes involving proton motion, these data are useful in identifying low-energy modes involving the amino substituents. These results are used in a more complete study of TATB's vibrational spectrum reportect later (SPP:3 below).

SPP:3. Vibration Spectra of 1,3,5-Triamino-2,4,6-Trinitrobenzene. B. L. Deopura and V. D. Gupta, J. Chem. Phys, 54, 4013-4019 (1970).

Complete infrared and Raman spectra of TATB are reported. A broad and diffuse inelastic neutronscattering spectrum is also presented (SPP:2). A Wilson GF matrix analysis is applied to the data to 
help assign the vibrational modes of the observed spectril resonances. This analysis results in the identification of a number of modes that appear at unusual vibrational energies relative to where these local frequencies occur in other molecules. Specifically, the N-H stretching frequency is much lower than in aniline, and even significantly lower than in paranitroaniline. This lower frequency is explained in terms of intra- and intermolecular hydrogen bonding. The out-of-plane wagging and inplane stretching frequencies of the $\mathrm{NO}_{2}$ and $\mathrm{NH}_{2}$ groups are also shifted as expected: All the results in this paper serve to support the idea of an extensive hydrogen-bonded system in the molecular and lattice planes. Note: This paper refers to TATB as TATNB (usually the abbreviation for 1,3,5-triazido-2,4,6-trinitrobenzene).

SPP:4. Vapor Pressure and Heats of Sublimation of Some High Melting Organic Explosives. J. M. Rosen and C. Dickenson, J. Cheim. Eng. Data. 14, 120-124 (1969).

The vapor pressure of TATB from $129^{\circ} \mathrm{C}$ to $177^{\circ} \mathrm{C}$ is reported, along with analogous data from RDX, HMX, TNA, DATB, and HNS. TATB is shown to have a high heat of sublimation, notably higher than that of DATB. Maximum vapor pressure achieved with TATB was $1.6 \times 10^{-5}$ at $177^{\circ} \mathrm{C}$. It is suggested that the layered, hydrogen-bonded structure of TATB adds to its stability as a solid material relative to the chain-like structure of DATB.

SPP:5. Properties of Chemical Explosives and Explosive Simulants. B.M. Dobratz, Lawrence Livermore National Laboratory, UCRL-51319 (1972).

The original appearance of $-36.85 \mathrm{kcal} /$ mole as the heat of formation of TATB in the LL,NL literature stems from work by C.'H. Shomate at the Naval Ordnance Test Station, China Lake (1970), but it appears that the details were never published. This report also presents the enthalpies of formation for a number of other explosives, determined by bomb calorimetry. The $\mathrm{H}_{\mathrm{f}}{ }^{\circ}$ for TATB of $-36.35 \mathrm{Kcal} / \mathrm{mol}$ is the value used in the LLNL Explosives Handbook.

SPP:6, Enthalpies of Formation and Calculated Detonation Properties of some Thermally Stable Explosives. P. E. Rouse, Jr., J. Chem. Eng. Data. 21, 16 (1976),

The heats of formation of a large number of explosive molecules, including TATB, were measured by bomb calorimetry. Work performed at Los Alamos reports the $\mathrm{Hf}^{\circ}$ of TATB to be $-33.4(1.2) \mathrm{kcal} / \mathrm{mole}$. This is the value used in Los Alamos Explosives Properties Data. It differs from tive LLNL value.

SPP:7. Growth of 1,3,5-Triamino-2,4,6,-Trinitrobenzene(TATB) 1. Anisotropic Thermal Expansion. J.R. Kolb and H.F. Rizzo; Prop. Exp. 4, 10-16 (1979).

$X$-ray crystallography is used to obtain the coefficient of thermal expansion in all three crystallographic directions. For pure, single crystals in the temperature range of $214 \mathrm{~K}$ to $377 \mathrm{~K}$, essentially all the expansion of the lattice occurs along the $c$ axis, increasing $5 \%$ in linear dimension over this temperature range. Not surprisingly, this axis corresponds to the direction perpendicular to the planar sheets of $\mathrm{H}$-bonded, linked TATB molecules in the $a-b$ plane. The expansion is reversible, Thermal expansion behavior is compared with that of two other planar crystallographic systems, boron nitride and graphite. Two other crystallographic phases are mentioned, a second triclinic with $\mathrm{Z}=1$ and a monoclinic form with $\mathrm{Z}=4$. Some doubt is raised as to whether the monoclinic form is simply a disordered form of the original triclinic lattice. Unfortunately, no additional work has been done on these polymorphs.

SPP:8, Free Radicals of TATB. A. D. Britt, W. B. Moniz, G. C. Chingas, D. W. Moore, C. A. Heller, and C. L. Ko, Prop. Exp, 6, 94-95 (1981).

Intense ultraviolet irradiation of TATB is shown to cause the formation of a stable paramagnetic species that exhibits a complicated ESR spectrum of more than 100 lines. The authors suggest that the signal originates from a free radical whose structure is very similar to TATB's, but one of the $-\mathrm{NO}_{2}$ groups has abstracted a hydrogen from a neighboring TATB molecule to form a $\mathrm{NO}_{2}-\mathrm{H}$ moiety. Curious hyperfine coupling constants are observed relative to what would be expected for a molecular orbital electronic structure that emphasizes an aromatic benzene ring. This is consistent with the distorted crystal structure (STR:1). 
SPP:9. The Sublimation of 1,3,5-Triamino-2,4,6-Trinitrobenzene (TATB). J. R. Kolb and R. G. Garza, Lawrence Livermore National Laboratory, UCRL-85971 (1981).

The authors investigated the details of the purity and yield from direct sublimation of TATB at temperatures as high as $200^{\circ} \mathrm{C}$. The sublimnte of TATB appeared as a bright yellow, polycrystalline material. Yields from small $(2.0 \mathrm{-g})$ samples were as high as $95 \%$. However, chemical analysis indicates that the impurities contained in the starting material are included in the sublimate. It is suggested that the material that sublimes is not individual TATB molecules but small clusters, $(\text { TATB })_{n}$. The first step in the sublimation process is loss of included water, with an estimated activation energy of $5.6(1.7) \mathrm{kcal} / \mathrm{mole}$. On the basis of small activation energy, the authors state that this is not water produced from a molecular dehydration reaction, as in the removal of two hydrogen atoms from the amino and one oxygen'atom to form a nitro group. This is simply water that is intercalated in the large space in the crystal lattice between the TATB planar sheets.

SPP:10. A Carbon Auger Line Shape Study of Nitroaromatic Explosives. J. W. Rogers Jr., H. C. Peebles, R. R. Rye, J. E. Houston, and J. S. Binkley, J. Chem. Phys. 80, 4513-4520 (1984).

Auger spectroscopy is used to indicate changes in hybridization at the carbon atoms in the $\mathrm{C}_{6}$ ring in a series of substituted nitroaromatics. Molecules studied included benzene, TNB (trinitro benzene), TNT (trinitrotoluene), MATB (monoaminotrinitrobenzene or picramide), DATB (diaminotrinitrobenzene), and TATB. Observations are interpreted in terms of the relative shock sensitivity of these compounds. Very subtle features of broad, experimentally noisy spectra are interpreted with great earnest. Arguments based on the data are presented to suggest that the electronwithdrawing properties of the nitro groups destabilize the carbon $\pi$-bonded ring system and therefore make nitro-substituted aromatics more sensitive to shock. However, the electron-donating characteristics of the amino group do not serve to compensate for the ring destabilization. Their contribution to the stabilization of the overall system is represented two ways. First, the donated electron density serves to create some $\pi$-like bonding between the carbon atoms and the amino groups and to increase the negative charge on the nitro groups, thus making the $\mathrm{NO}_{2}$ groups much more negative and the $\mathrm{NH}_{2}$ groups appear more positive. This leads to the second point, asserting that this enhanced polarization dramatically increases the amino-substituted nitrobenzene's capability for intermolecular $\mathrm{H}$ bonding. Although amino substitution does not strengthen the $\mathrm{C}_{6}$ ring, it still stabilizes these molecules due to these other consequences.

SPP:11. Vibrational Spectrum of 1,3,5-Triamino-2,4,6-Trinitrobenzene. T. G. Towns, Spectrochim. Acta 39A, 801-804 (1983).

The infrared spectrum of TATB, D6-.TATB, ${ }^{15} \mathrm{NO}_{2}-\mathrm{TATB},{ }^{15} \mathrm{NH}_{2}-\mathrm{TATB}$, and ${ }^{15} \mathrm{NO}_{2},{ }^{15} \mathrm{NH}_{2}-\mathrm{TATB}$ is reported in the 400 to $4000 \mathrm{~cm}^{-1}$ region. Some of the assignments for nitro and amino modes in the 1000 to $1300 \mathrm{~cm}^{-1}$ region disagree with those of Deopura and Gupta (SPP:3). The $15 \mathrm{~N}$ substitution doesn't help mach, but the $2 \mathrm{H}$ spectrum clearly supports the assignments given here, particularly for several amino inodes. These assignments are discussed in terms of partial changes in bond order for the ring carbon-substituent nitrogen bonds and intramolecular hydrogen bonding.

SPP:12. Structure Moleculaire des Derives Nitres Aromatiques \#5-Spectres de Vibration et Analyse par Coordonnees Normals du TATB. (Molecular Structure of Nitro Derived Aromatics, No. 5-Vibrational Spectra and Normal Coordinate Analysis of TATB). (French, abs. in English). G. Vergoten, G. Fleury, M. Blain, and S. Odiot. J. Raman Spect. 16, 143-148 (1985).

This paper reports the infrared and Raman spectra of TATB over the range of 10 to $4000 \mathrm{~cm}^{-1}$. It does not differ much from those of Deopura and Gupta (SPP:3 above), save for the addition of very-lowenergy modes to the data set. It questions the assignments of Towns (SPP:11) based on the lack of Raman data and the density of the spectra in the 1600 to $700 \mathrm{~cm}^{-1}$ region. However, this work does not include any isotopic substitution. The authors do a Urey-Bradley force-field calculation (more sophisticated than Deopura and Gupta's), and a number of the spectral features are reassigned differently from those in Towns or in Deopura and Gupta. The calculation, however, clearly indicates that many of the vibrations in this molecule are not well described by a single coordinate such as $\mathrm{NH}_{2}$ bend or $\mathrm{NO}_{2}$ 
symmetric stretch. Many molecular vibrations couple fairly strongly with one another. As a consequence, direct interpretation of the positions of these vibrational levels, and attempts to relate them to the complicated electronic structure of this molecular system, including the hydrogen-bonded network, are a little dangerous.

\subsection{Technical Summary}

The crystal-structure paper by Cady and Larson (1965) opened the door to a number of other works that rely on a knowledge of intrainulecular dimensions, particularly the three vibrational spectroscopic papers and the ab initio calculations reported in the Theory section below. The spectroscopic papers are in general agreement with regard to the amino features. Most notable is the fact that the $\mathrm{N}-\mathrm{H}$ stretching frequency in TATB is unusually low due to hydrogen bonding. Additionally, out-of-plane motions by $\mathrm{NO}_{2}$ and $\mathrm{NH}_{2}$ groups show increased frequencies due to the hydrogen bond linking neighboring groups together. Complete assignment of the $\mathrm{C}-\mathrm{NO}_{2}$ modes is very difficult due to strong mixing with carbon ring modes. The overwhelming structural features of TATB are intra- ald intermolecular hydrogen bonding, which contributes to the graphitic-like packing of molecular sheets within the crystal. Many physical properties of TATB are related to this characteristic, including thermal expansion properties and heat of sublimation.

\section{Differential Thermal Analysis and Unconfined Decomposition (DTA)}

\subsection{Overview}

Thermal analysis, which is based on a variety of techniques, involves carefully measuring energy absorbed by or released from a material as temperature is varied. In the study of energetic materials the relationship between rates and energy as a function of temperature has been used to identify different reaction'stages, Arrhenius kinetic parameters, and phase changes. Although most thermal decomposition reactions in the solid state are very complicated, some useful information can be extracted. The major pitfall associated with this type of work is overinterpretation of the results.

This techrique was first pioneered on energetic materials by R. Rogers of Los Alamos in 1972, when he began to examine the behavior of decomposition exotherms in HMX and RDX. The general technique dates back to the mid 1960s. Rogers conducted several other studies and continued to advance the methods of interpreting the data. However, concer as over which regimes in the exothermic behavior of a given sample can be interpreted in terms of Arrhenius kinetics have continued to hamper attempts to relate exothermic behavior to a specific, identifiable reaction step.

In the late 1970s and early 1980s, S. Zeman in Czechoslovakia conducted a set of differential thermal analysis (DTA) studies on a wide range of energetic materials, including polynitroaromatics. An attempt was nade to relate these results to a wide variety of explosive properties (time to explosion, detonation velocity, etc.). Although these relationships appear to be somewhat dubious, some insight can be gained by handling the data presented in these papers in a less aggressive fashion. The papers reference an enormous body of work in the Soviet literature examining thermal decomposition of energetic materials. 


\subsection{Individ kal Studies}

DTA:1, uimplified Determination of Rate Constants by Scanning Calorimetry, R, N. Rogers, Anal. Chem1. 44, 1336-1337 (1972).

This paper briefly describes Rogers's method for determining first-order rate constants from differential scanning calorimetry (DSC) data. It doesn't report anv work on TATB; however, it is useful to examine if later DSC work is to be studled.

DTA:2. Determination of Condensed-Phase Kinetics Constants. R. N. Rogers, Thermochim. Acta. 9, 444-446 (1974).

The DSC curve of RDX is examined in detail. TATB is not addressed, but this paper is also very instructive regarding the techniques and analysis problems associated with DSC experiments in the condensed phase. It points out how vapor-phase reactions can be separated from the condensed-phase contribution to the overall exotherm. The paper shows how a number of DSC experiments at different temperatures can be used to determine Arrhenius kinetic parameters.

DTA:3. Thermal Stabilities of Polynitroaromatic Compounds and Their Derivatives. S. Zeman, Thermochim. Acta. 49, 269-283 (1979).

This is the first in a series of three papers by Zeman attempting to relate non-isothermal DTA results to detonation properties in a wide range of energetic nitro-substituted compounds. Thirty-three compounds were examined, including TATB. Two properties, $T_{\max }$ and $\mathrm{T}_{\mathrm{D}}$, are defined and calculated

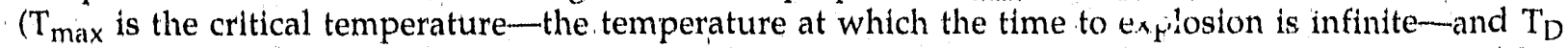
is a value obtained from the DTA experimental exotherm), Experimental results are compared with values of these parameters calculated from Arrhenius parameters ( $E$ and $A$ ) obtained from the Soviet literature. DTA behavior was also examined for many of these compounds when mixed and/or dissolved in trinitrobenzene (TNB). In a lengthy discussion of the results, a few observations are notable regarding TATB. First, it is stated that TATB is slightly less thermally stable than MATB or DATB, which are very similar in their behavior when in a TNB solution. Second, all aminosubstituted TNBs are less stable than TNB itself. However, pure solid TATB is more stable than solid MATB or solid DATB, and is only a little less stable than TNB. If the amino-substituted species fully dissolve in TNB as individual molecules, then these data tend to support the contention that TATB's lattice serves as a thermally stabilizing environment.

DTA:4. The Relationship Between Differential Thermal Analysis Data and the Detonation Characteristics of Polynitroaromatic Compounds. S. Zeman, Thermochim. Acta. 49, 199-212 (1980).

This paper, the second of a series, uses the data from DTA:3 to relate DTA T $D$ s to Chapman-Jouguet pressures and detonation velocities. The author uses a wholly empirical relationship to fit 37 explosives gathered into six "similar-structure" groups analyzed in two different ways. The members of any given "structure group" are claimed to possess common properties such as $\mathrm{sp}^{2}$ hybridization of an amino group, as is the case in TATB's group. The use of these groupings to generate a correlation between $P_{C J}$ and DTA results see!ns forced. Fourteen of the 37 compounds studied are not included in any of the analyses, perhaps because they didn't fit any correlation. The compounds that are used are broken into sufficiently small groups that eight parameters are used to fit 21 observations. This does not represent a particularly good correlation. It appears as though the molecules may have been grouped after the analysis rather than before. This is an unfortunate example of overinterpretation of a technique that has useful but limited applicability. The results of the first paper (DTA:3) are fairly informative, but there is no reason for the onset temperature of the lowest exotherm to be correlated directly with overall energy release of the material. 
DTA:5. Kinetic Data from Low-Temperature Thermolysis in the Study of the Microscopic Initiation Mechanism of the Detonation of Organic Polynitro Compounds. S. Zeman, Thermochim. Acta 49, 219-246 (1981).

This long paper is the third and final edition of three papers by Zeman relating DTA properties of explosives to detonation properties. Seventy-four compounds are examined. There is a very long discussion section in which detonation velocities are related to reaction rate constants and activation energies (see DTA:4). The author uses these dublous relationships to discuss actual reaction mechanisms for different groups of nitro compounds, including nitramines, nitre alkanes, alkyl nitrates, and polyntitoaromatics. Several statements are made concerning TATB and its group of aminosubstituted polynitroaromatics. The paper shows many plots of these relationships. From the graphical representations it is fairly evident that the correlations here and in DTA:4 are weak at best. The author states that Rogers's DSC results and those in this paper indicate that the primary fragmentation mechanism of DATB and TATB differs from the mechanism for other polyamino polynitroaromatics. The entropy of activation favors a primary homolytic mechanism such as the fission of the $\mathrm{C}-\mathrm{NO}_{2}$ bond as the reasonable assignment of the initial thermal decomposition mechandsm in both the solid state and in solution. It is also pointed out that although additional thermal stability in TATB may originate from its unique lattice structure, this stability is "conditioned" by the structure of the molecule and is not a property of the general group. That is to say, the paper emphasizes that TA'TB's thermal stability originates primarily from single-molecule properties and not from solid-state lattice effects. This paper references a wealth of experiments on TATB and other nitroarenes in the Soviet literature.

DTA:6. Thermal Decomposition of TATB. M. Farber and R, D. Srivastava, Combustion and Flame 42, 165-171 (1981).

The products of thermal decomposition during sublimation of TATB are analyzed by mass spectrometry. Considerable effort was made to use ionizing electrons at as low an energy as possible to minimize fragmentation in the spectrometer. Two different thermolysis methods, effusion and Langmuir, were used. The authors felt that one of the methods, the effusion method, contained too many gas-phase decomposition products; thus their discussion dwells on results from the Langmuir method alone. The most significant result is that large peaks were observed, corresponding to ring cleavage reactions producing $C_{3}$ species with accompanying nitro or amino groups. There was some evidence for loss of $\mathrm{NH}_{2}$ from the $\mathrm{C}_{3} \mathrm{~N}_{3}$ fragments. There was no evidence of simple nitro loss from TATB itself. This finding disagrees with the conclusions of DTA:7 below. More recent calculational work (see Theory section) is consistent with $\mathrm{C}-\mathrm{NO}_{2}$ bond rupture as being the initial and important decomposition pathway and does not agree with this proposed ring fission.

DTA:7, X-Ray Photoelectron Study of Electronic Structure and Ultraviolet and Isothermal Decomposition of TATB. I. Sharma, W. L. Garrett, F. J. Owens, and V. L. Vogel, J, Phys. Chem. 86, 1657-1661 (1982).

The photoelectron spectra of TATB, UV-exposed TATB, and partially thermally decomposed TATB are examined in the valence region and in the core regions of nitrogen, carbon, and oxygen. The positions of various binding energies are compared with those of DATB, picramide (MATB), and trinitrobenzene to aid in the interpretation. Low-level calculations (CNDO-MO) are also used to help interpret the data. The authors state that the evidence indicates that UV photolysis primarily serves to break the $\mathrm{C}-\mathrm{NO}_{2}$ bond. Thermal decomposition also severs $\mathrm{NO}_{2}$ from the ring, but there is also evidence that the $\mathrm{NH}_{2}$ group is affected to some extent. It is suggested that this may be due to loss of $\mathrm{H}^{+}$or that the changes in the amine nitrogen peak are due to a change in the interaction of the amino group with neighboring functionalities in the lattice. The direct loss of $\mathrm{NO}_{2}$ from the TATB ring during thermal decomposition is contrary to the mass spectroscopy observations in DTA:6 above. 
DTA:8. An Enthalpic Study of the Thermal Decomposition of TATB. E. Catalano and P. Crawford, Thermochim. Acta. 61, 23-36 (1983).

This is the first in a series of three back-to-back papers in Thermochimicn Acta by Catalano et al. dealing with the thermal decomposition of TATB. The first is listed here. The second and third are in the Time to Explosion section below. This paper is a detailed DSC study of unconfined TATB that shows that as many as five different reaction stages are observable in a single temperature scan. Several conclusions are drawn. Probably the most important point is that in the range of 528 to $630 \mathrm{~K}$ there are no detectable endothermic steps despite the suspicion that the thermal decomposition of TATB might start with such a step. There is no evidence of melting or vaporization. An effort was made to use kinetic theory to analyze the different reaction regions; however, the authors express justifled concern over the actual applicability of such theory to a complicated reacting environment that is in the solid phase and does not appear to be first-order. The best fit to the data suggests that the initial decomposition step is autocatalytic. The lack of an observable endothermic stage of vaporization is rationaltzed by suggesting that such a stage occurs concurrently with the exothermic decomposition. The paper frequently references private communication with $\mathrm{H}$. Cady and J. Janney of Los Alamos regarding work that appears to be very similar to that reported here. A paper cliscussed later (TTX:9) by Rogers, Janney, and Ebinger, published a year earlier, also suggests an autocatalytic decomposition mechanism.

\subsection{Technical Summary}

Non-isothermal differential thermal analysis and isothermal differential scanning calorimetry have been used on solid samples of TATB and related compounds by a number of different workers in the field. This work reveals several important aspects of the molecule's unconfined thermal decomposition. Some of these results are fairly quantitative, such as Arrhenius parameters that can be inserted into Frank-Kamenetskil relationships or computer time-to-explosion models. A certain amount . of more qualitative information can also be gathered, such as the identification of distinct reaction regimes that may not lend themselves to quantitative analysis but can still be used to determine endothermic vs exothermic steps, reaction order, and reaction regimes that are likely to be associated with rapid energy release.

The prospect of using Arrhenius parame ers obtained in the DSC to predict times to explosion or critical temperatures is very attractive. These efforts reflect a fairly rare opportunity in the overall field of energetic material research to use an independent measurement technique to obtain quantitative predictions about a more or less unrelated explosive response property. The unfortunate aspect of these efforts is that all the models require additional information about the sample that is not measured in the DSC and is not known accurately. As a consequence, the "Arrhenius parameters" obtained are simply a part of a greater set of parameters, many of which can be seen to be, in effect, adjustable. This circumstance degrades the predictive capability of these models.

Several mass-spectroscopy studies have identified authentic reaction products, but there are difficulties and contradictions associated with connecting sets of products to specific reaction stages. The mass spectroscopy results of DTA:6 do not show any $\mathrm{NO}_{2}$ as an initial thermal decomposition product. This contradicts much of the photoelectron spectroscopy and inuch of the theoretical/ computational effort discussed later in this review.

There is a possible explanation for this discrepancy. The photoelectron work examines the nature of the condensed-phase residue. It is not likely that small molecules, such as $\mathrm{C}_{3}$ fragments, would be found in the solid phase to begin with. The mass-spectrometric work examines gas-phase products from a heated solid. Since TATB is know'n to sublime with some decomposition, these mass spectra in DTA:6 may simply represent the products of gas phase decomposition of sublimed molecules. These results may have little bearing on condensed-phase reactivity. Photoelectron spectra can probably identify changes in composition (that is, loss of starting material), but it is unrealistic to suggest that a particular molecular species can be identified with this rather coarse analytical technique. 
Essentially, neither experiment produces definitive results. TATB may very well have a completely. different reactivity in the condensed phase relative to pure gas-phase decomposition.

\section{Time to Explosion and Confined Pyrolysis (TTX)}

\subsection{Overview}

Time-to-explosion (TTX) experiments have been conducted since the early 195Us. The original experiments were conducted by Henkin and MoGill. In the sixties and early seventies, C. Mader, J. Zinn, R. Rogers, and others at Los Alamos used a modified version of the Henkin experiment to test the applicability of the long-standing Frank-Kamenetskii critical temperature relationship to some nitramine explosives. The results of these experiments, however, were dependent on the geometry and size of the sample. TATB was not addressed in most of these earlier studies. In the mid 1970s the technique was extended at LLNL by R. McGuire, E. Catalano, and others in a new cell design that provided for easier computer modeling, called a one-dimensional time-to-explosion (ODTX) experiment. This work resulted in thermal decomposition models for a variety of explosives, including TATB. It raised many questions about the importance of the role of confinement in a variety of thermal response properties of energetic materials. In the bulk of this work, results on TAT"B are combined, primarily in survey studies along with other common explosives. Only a few papers direct much discussion solely at TATB.

Included in this section of primarily ODTX experiments are results of critical-temperature measurements. Although a popular concept in the 1960s and earl; 1970s, it was essentially replaced by the more detailed ODTX experiments, which couple well with con puter modeling based on finiteelement heat-flow programs.

\subsection{Individual Studies}

TTX:1. Thermochemistry of Explosives. R. Rogers, Thermochim. Acta 11, 131-139 (1975).

This work recognizes one of the main problems posed by the concept of a critical temperature $\left(T_{c}\right.$, the temperature below which a confined sample will not explode) for an HE. The problem is that $T_{c}$ is dependent on sample shape and size. In addition, the author recognizes that the Frank-Kamenetskii relationship, together with an infinite set of kinetic parameters (pre-exponential and activation energy), can generate the correct critical temperature for a given size of HE. This paper combines DSC with critical temperature measurements to determine the "correct" set of parameters that will predict the $T_{c}$ of an explosive of any size or shape. Ten different explosives are studied, including TATB. In general, the DSC parame:ers do a pretty good job for most explosives in this study, reproducing the known critical temperature in the Franck-Kamenetskii equation fairly accurately. The analysis incorporates a "semi-adjustable" parameter, $Q$, the heat of the reaction ascribed to the initial isotherm. This parameter provides about $15^{\circ} \mathrm{C}$ worth of a fudge factor for fine-tuning the results. The paper asserts that time to explosion is very difficult to calculate from DSC results, since it is determined mostly by slow, solid-state processes. Critical temperature is primarily determined by the rapid reactions that follow slow decomposition and is sampled accurately by DSC. TATB is singled out as an example of a system that decomposes entirely in the solid state. Despite this paper's weaknesses, it is a pioneering attempt to use an independent chemical measurement, DSC, to predict another property, critical temperature, through a theoretical relationship. 
TTX:2. Henkin Time to explosion for TATB. L.C. Myers, Mason-Hanger Silas, MHSMP-77-18H (1977).

This report concerns itself with the reproducibility of TTX experiments (Henkin-like, using an aluminum blasting cap) in different batches of TATB. It is shown that within a given lot, the time to explosion at $367^{\circ} \mathrm{C}$ is reproducible to about $15 \%$. However, different lots of TATB can vary from an average TTX of $8.8 \mathrm{~s}$ for dark-colored TATB to as high as $200 \mathrm{~s}$ for a sample recrystallized from biphenyl. It is surprising that this enormous fluctuation from lot to lot is not discussed in more detail in other, later TTX studies. The paper raises an important question: if only a few percent of impurity can change the TTX wildly, then how can any chemical model based solely on TATB properties be expected to reproduce TTX results for formulated explosives?

TTX:3. The Thermal Decomposition and Reaction of Confined Explosives. E. Catalano, R. McGuire, E. Wrenn, D. Ornellas, and J. Walton, Sixth Symposium (Int.) on Detonation, 214-222 (1977).

This is the initial paper in the series of ODTX studies conducted at LLNL from 1975 to 1983 . It explains the advantages of this technique over those used in previous experiments. The paper reports many important results: (1) Confinement of the sample (whether or not product gases from early decomposition reactions can escape) plays a very important role in TTX results. (2) In agreement with Rogers (TTX:1), the Arrhenius constants derived from DSC measurements are not very good for TTX results. (3) In general, DSC results are not valid for predicting critical temperature in confined environments (this is very important). Finally, (4) Laplace-equation analysis of the heat flow and a single heat-source term cannot repreduce the experimental TTX results. This last result conflicts with the conclusions in TTX:4, which claims the success of a single reaction and heat-source model in modeling TATB's TTX behavior. In the Catalano et al. paper, however, several two-stage reaction schemes, which can be handled easily by a computer, do a much better job of representing the data. Specific behavior of TATB is not discussed.

TTX:4. Thermal Response of Explosives Subjected to External Heating. A. Popolato, J. J. Ruminer, A. S. Virgil, N. K. Kernodle, and D. L. Jaeger, Los Alamos National Laboratory, LA7667-MS (1979).

This report represents another early attempt to model TTX results with a heat-flow/heat-source computer code. The code, EXPLO, calculated temperature within a sample as a function of time and space. The chemical kinetics scheme used was very simple, employing a first-order reaction model. A number of explosives were examined, including TATB, all of which were successfully modeled to within $5 \%$ except for plastic-bonded NQ (nitroguanidine).

TTX:5. The Thermal Decomposition of Explosives with Full Containment in OneDimensional Geometries. C. M. Tarver, R. R. McGuire, E. L. Lee, E. W. Wrenn, and K. R. Brein, Seventeenth Symposium. (Int.) on Combustion, 1407-1413 (1978).

This paper is a continuatior of the ODTX experiments presented at the 6th Detonation Symposium. (TTX:3 above). It places more emphasis on experimental results and computer modeling than on the experimental apparatus. A three-step kinetic model is shown to calculate successfully the observed behavior of a number of explosives, including TATB. The authors emphasize the need for more accurate values of the confined material's thermal conductivity and heat capacity at elevated temperatures. TATB is singled out with two specific observations. First, in contrast to any of the other explosives examined, the addition of void space to the sample does not affect either the critical temperature or TTX of TATB. Second, TATB produces only low-order events when it "explodes." Low-order events characteristically do little or no damage to the confining apparatus. HMX-based explosives typically exhibit much mote violent behavior. It should be noted that the three-step kinetic model contains nine parameters, four of which are locked to the values reported by Rogers (TTX:1) for the fast reaction steps. 
TTX:6. Chemical Decomposition Models for the Thermal Explosion of Confined HMX, TATB, RDX, and TNT Explosives. R.R. McGuire and C. M. Tarver, Seventh Symposium (Int.) on Detonation, 56-63 (1981).

This paper follows TTX:5 in the LLNL series of ODTX experiments. A number of pure explosives are examined (including TATB) as well as several formulations that include RX-26-AF, a mixed (HMX, TATB) formulation. Actual chemical reactions-specifically, ring depolymerization followed by fragmentation-are suggested for RDX and HMX decomposition, along with a refined reaction-step model for these pure materials. The authors interpret TNT as having a different reaction sequence from RDX and HMX, with the same form as that of TATB but different parameters. They use a three-step autocatalytic process with unidentified reactions. The authors again emphasize the need for accurate thermal conductivity data. The refinement of the reaction models for these different explosives and formulations permits much more accurate modeling thain in the earlier publications (TTX:3 and TTX:5 above). Calculations for electron-beam heating are reported (see TTX:7). The models for PBX9404 and TATB do not perform as well in these electron-beam experiments as in the LLNL ODTX experiments, but this can probably be ascribed to the lower containment threshold (less than $250 \mathrm{~atm}$ maximum pressure vs $1500 \mathrm{~atm}$ for the LLNL ODTX tests) in the electron-beam experiments. The model for TATB is based on an initial slow endothermic first step, followed by a pair of rapid, very exothermic steps. The authors report that a large amount of "solid reactive intermediate(s)" (not otherwise identified) are produced before the exothermic process takes over.

TTX:7. Electron Beam Initiation of High Explosives. A. Stolovy, J. B. Aviles Jr, E. C. Jones, Jr., and A. I. Namenson, Seventh Symposium (Int.) on Detonation, 50-55 (1981).

This paper represents a significant advance in TTX experiments. An electron beam is used to uniformly heat a sample of high explosive containing thermocouples and pressure sensors. As these sensors had a $2-\mathrm{ms}$ time resolution, pressure and temperature histories were obtained simultaneously as the sample decomposed. Confinement was maintained up to $4000 \mathrm{psi}$. The study examined TATB, $\mathrm{HBX}-1$, and PBX 9404. The most important result is that a multi-step reaction model is clearly needed to describe the thermal decomposition of TATB. Two exothermic reaction stages occur during the rapid. electron-beam heating. The initial weak exotherm is followed by a flattening of the temperature curve before a large exotherm leads to explosion. At least two exotherms, with two very different rates, are present. TATB results in this study are presented in much more detail in TTX:9.

TTX:8. Kinetic Isotope Effect in Thermal Explosions. R. N. Rogers, J. L. Janney, and M. H. Ebinger, Thermochimica Acta 59, 287-298 (1982).

This paper reports on a combination of differential scanning calorimetry and TTX experiments on $H^{6}$ and $D^{6}$ TATB, $H^{8}$ and $D^{8}$ PETN, and $H^{2}$ and $D^{2}$ DINGU. It points out that deuteration of specific functional groups can be used to identify elementary reactions that occur during the complex process of overall thermal decomposition of an explosive. Results for fully deuterated TATB are interpreted in detail. The authors state that, on the basis of the difference between measured DSC activation energies for $D^{6}$ and $\mathrm{H}^{6} \mathrm{TATB}$ and the difference in critical temperature between these two molecules, they have properly identified the kinetic constants associated with the regime of "consistent reaction" that is responsible for self-heating to explosion. The $\mathrm{k}-\mathrm{H} / \mathrm{k}-\mathrm{D}$ ratio of 1.5 indicates the presence of a primary deuterium isotope effect. This initial early-time reaction is identified as autocatalytic and involving the amino groups. Evidence is presented that late-time reactivity is identical for $\mathrm{H}^{6}$ and $\mathrm{D}^{6}$ TATB, suggesting that the reactive molecules are devoid of hydrogen at late times.

TTX:9. Exothermic Reactions in TATB Initiated by an Electron Beam. A. Stolovy, E. C. Jones Jr., J. B. Aviles, Jr., A. I. Namenson, and W. A. Fraser, J. Chem. Phys. 78, 229-235 (1983).

This paper expands on the preliminary results discussed in TTX:7 above. The authors deal exclusively with TATB. They examine the exotherms of both confined and unconfined samples subjected to rapid heating by an electron beam. During heating of unconfined TATB (about 5 to 10 seconds at these dose rates), the temperature of the sample rises linearly to a plateau at $450^{\circ} \mathrm{C}$. The authors assume that'this plateau is due to the melting of the TATB sample. Latent heat of fusion is estimated to be 
$26 \mathrm{kcal} / \mathrm{mole}$. The plateau is followed by an exotherm. Confined samples were shown to exhibit very different behavior. Because the sample is gauged with thermocouples, exotherms can be observed as a function of sample temperature and not simply duration of heating. A number of different reaction regions are observed when the data are analyzed in this way. Remarkably, the values of several kinetic parameters extracted from the data in a region of "consistent reaction" (TTX:8) agree with those obtained by Rogers (TTX:8) in a significantly different experiment. Similar values of kinetic parameters are obtained for a TATB/Kel-F 800 formulation. In discussion, the authors observe that above $395^{\circ} \mathrm{C}$ the thermal decomposition of confined TATB is "certainly non-Arrhenius," but that there are regions where "effective kinetic constants" can be assigned. The chemical significance of these values is not at all clear. The paper defers to Rogers et al. (TTX:8) for much of the significance of its results for chemical reactivity.

TTX:10. A Study of the Thermal Decomposition of TATB. The Gaseous Products and Kinetics of Evolution. E. Catalano and C. E. Rolon, Thermochim. Acta. 61, 37-51 (1983),

This is the second of a series of three papers by Catalano et al, on the thermal decomposition of TATB (the first paper is in the Thermal Analysis section, the third is described below). The products generated from thermal decomposition of TATB confined up to 10,000 psi were analyzed by mass spectroscopy and by matrix-isolation, Fourier-transform infrared spectroscopy. Evolving product gases were selected in aliquots at different times during the decomposition reaction and correlated with the internal pressure of the reaction vessel and with time of reaction. Pressure-vs-time plots of the reacting sample suggest an early endothermic stage followed by a transition to what the authors interpret as an exothermic reaction, still prior to explosion. The products are analyzed in terms of these two stages. Early-time reactions yielded primarily $\mathrm{CO}_{2}, \mathrm{H}_{2} \mathrm{O}$, and $\mathrm{N}_{2}$. No NO was observed during the endothermic stage but was seen at longer times. "Endothermic" products are interpreted as simply the removal of intercalated molecules in the TATB lattice, not reaction of TATB itself. The authors state that their evidence does not support the abstraction of intramolecular water for the TATB molecule, as: suggested by G. Andrews at a 1977 ERDA (Energy Research and Development Administration) compatibility meeting.

TTX:11. On the Solid State Products of the Thermal Decomposition of Confined and Unconfined TATB. E. Catalano and C. E. Rolon, Thermochimica Actn 61, 53-71 (1983).

This is the third in a group of three back-to-back papers on TATB published in Thermochimica Acta. The paper is presented as a preliminary study of solid producis left after explosion of TATB samples in ODTX experiments. The products are examined by infrared spectroscopy, optical microscopy, and scanning electron microscopy (SEM). The samples, composed of a variety of different materials resulting from different levels of confinement, ranged from crystalline solid to a deposit that appears more amorphous. Infrared spectral differences between the TATB starting material and the different solid products are small but notable. Optical and SEM photographs show that unconfined decomposition yields a variety of solid-state products. The authors describe a reaction scheme in which three products occur at three different temperatures and use it to interpret the infrared data. Lower-temperature products appear still to contain $\mathrm{N}-\mathrm{H}$ and $\mathrm{N}-\mathrm{O}$ bonds. Higher-temperature products are, believed not to contain any $\mathrm{N}-\mathrm{H}$ bonds. Although this work is identified by the authors as preliminary, we found no published follow-up work.

TTX:12. Comparative Study of Molecular Fragmentation in Sub-Initiated TATB Caused By Impact, U.V., Heat, and Electron Beams. J. Sharma, J. C. Hoffsommer, D. J. Glover, C. S. Coffey, F. Santiago, A. Stolovy, and S. Yasuda, Shock Wares in Condensed Matter-1983, pp. 543-546.

$X$-ray photoelectron spectroscopy (XPS) and chemical ionization mass spectroscopy were used as diagnostic tools to identify different chemical species present in residues produced when TATB responds If different stimuli. Thin-layer chromatography was used to separate several different pure products from the residue. Material from all forms of stimuli displayed photoelectron spectra indicative of samples that have lost their $\mathrm{NO}_{2}$ functionalities and show nitrogen in a diff rent oxidation state than in TATB. Mass spectrometry identifies parent-ion masses consistent with monofurazan and furoxan structures that originate from loss of water, $\mathrm{NO}_{2}$, or hydrogen. The authors suggest that the first 
endothermic step in the decomposition of TATB is loss of aromaticity that is endothermic (but sensitizing), followed by an exothermic step that involves the loss of $\mathrm{H}_{2} \mathrm{O}$ from the starting material. Under optical and SEM examination, selected TATB residues appeared very similar whether produced by electron-beam heating or by less exotic thermal decomposition. Products of drop-hammer detonation and UV photolysis were similar, consisting of a greater loss of nitro groups and little or no production of furcoxans.

\subsection{Technical Summary}

Time-to-explosion and critical-temperature measurements have two basic purposes, depending on how an experiment is interpreted. The most conservative way of interpreting TTX experiments is to view them simply as safety tests. That is, they give a handler of a particular explosive a "ball-park" idea of the thermal stability of the material concerned. The second use, the one emphasized here, is that for a given geometry, sample size, level of confinement, and heating temperature, TTX is a fundamental property of an explosive. It is highly reproducible from test to test and can be directly related to the complex chemical reactions present during thermal ignition.

No single equation and set of model parameters can calculate a TTX for any given material. However, more complicated computer models using a single set of paramiters can accurately reproduce TTX curves over wide temperature ranges. The work of Tarver and McCulre shows quite clearly the high level of precision of which these models are capable. More important, the model parameters can be determined from small-scale tests and are transferable to other geometries and sample sizes. However, they rely on simple, $A$ reacts to $B, B$ to $C$ and $D$, etc., generalized reaction schemes and cannot be used to identify specific reaction coordinates or possible products. As a consequence, a successful model appiicable to one explosive provides little chemical insight as to how a different, but chemically related, system might behave. The next logical step is therefore to analyze reaction products and intermediates, and where possible, to correlate model reaction steps with real chemical events. This step points to the work of Catalano et al. that follows up TTX modeling done at LLNL in the 1970s.

Specific results for TATB to date are as follows. For confined geometries, there appears to be a slight endothermic step preceding the major energy-releasing stage of the lecomposition of the bulk material. Confinement does not affect time to explosion, as is the case with most other explosives. A most important point, however, is that despite so many different sample reclamation studies using a variety of analytical techniques it is still not clear what small molecules are produced at what stages in the thermolysis of TATB.

\section{Shock Response (SHK)}

\subsection{Overview}

The shock response of an explosive as widely used as TATB in its common formulations, LX-17 and PBX-9502, has, of course, been studied in considerable detail. The bulk of the work has been phenomenological. That is to say, most of the detailed studies done throughout the 1970s were designed simply to categorize TATB formulations relative to other widely used explosives in a variety of safety tests (drop-hammer test, skid test, Susan test, booster tests, gap test, and failure diameter tests, to name a few). Little interpretation of the molecular chemistry or structure of the TATB lattice is offered in this literature (or is necessary for these tests to serve their intended purpose). Much of this work is collectea in B. M. Dobratz, M. Finger, L. G. Green, J. R. Humphrey, R. R. McGuire, and H. F. Rizzo, Selected Sensitivity Tests of TATB and TATB Formulations and their Evaluation, Lawrence 
Livermore National Laboratory, UCID-18026 (1979). This compenditum is required reading for anyone interested in the detalls of TATB's sensittvity virtues.

The purpose of this review is to supply information on work that attempts to attribute TATB's insensitivity in terms of its chemical make-up. As a consequence, we will not discuss here the extensive literature so well summarlzed in the LLNL Explosines Handbook and in Los Alamos Explosives Properties Data. There are, however, several interesting studies that do relate measurements on shocked TATB to its chemical reactivity. These are reported below.

\subsection{Individual Studies}

SHK:1. The Hydrostatic Compression of Explosives and Detonation Products to $10 \mathrm{GPa}$ and Their Calculated Shock Compression: Results for PETN, TATB, CO2, and H2O. B. Olinger and H. Cady, Sixth Symposium on Detonation, pp, 700-709 (1977).

Using powder $x$-ray diffraction techniques and Bridgman anvils (tungsten carbide), the authors determined the isothermal compressibility of TATB. This was the first determination of the onedimensional nature of the high-pressure responses of TATB. Early in the paper it is suggested that this information can be used to better understand the shock-induced reactivity explosives. The shock - Hugoniot of TATB is calculated from isothermal compression data and other known physical constants. There is little comment on the similarities and differences between calculated and measured Hugonlots.

SHK:2. XPS Study of UV and Shock Decomposed Triamino-trinitrobenzene. J. Sharma and F. J. Owens, Chem. Phys. Lett. 61, 280-282 (1979).

The x-ray photoelectron spectrum of TATB exposed to ultraviolet (mercury lamp) radiation is compared with that of shocked TATB samples. The shock intensity was well below what would initiate a detonation. The spectral region used for comparison corresponded to that produced by electrons emitted from the $1 \mathrm{~s}$ orbital of nitrogen atoms, incliding both the amino and the nitro substituents. UV photolysis is shown to reduce the intensity of the nitro is peak relative to the amino nitrogen 1 s peak by as much as $30 \%$. The shocked sample showed a trend in the same direction but displayed a much weaker effect. The authors conclude that photolysis serves to break $\mathrm{C}-\mathrm{NO}_{2}$ bonds. They also conclude that this is the primary effect of a weak shock. That is to say, the first step in shock-induced decomposition of TATB is the rupture of the $\mathrm{C}-\mathrm{N}$ bond of one of the carbon ring's nitro groups. The authors may have stretched their shock data somewhat. In the figure presented in the paper, the shocked and unshocked spectra appear identical. The stated nitro/amino intensity ratio is 0.899 for unshocked TATB and 0.84 for the shocked sample; however, the figure cannot show a $5 \%$ change in the ratio of the intensities of the two peaks. Whether this $5 \%$ change is present or not, it is dangerous to try to interpret such a small effect.

SHK:3. Detonation Properties of TATB when Impacted by Hypervelocity Projectiles. J. Delistraty and H. Brandt, Propell. Expl. Pyrotech. 7, 113-122 (1982).

The authors analyze in detall four gun experiments on the shock sensitivity of TATB. The results are used to support the validity of Vector HEMP, an LLNL computer program (hydrodynamic code) that calculates pressures and detonation characteristics using a reactive flow. The experimental results show that a $3.06-\mathrm{km} / \mathrm{s}$ steel rod possesses insufficient shock energy to initiate TATB. A shot at 4.75 $\mathrm{km} / \mathrm{s}$ results in localized ignition of the sample but no high-order detonation. A steel rod at $5.67 \mathrm{~km} / \mathrm{s}$ and a tantalum plate at $6.53 \mathrm{~km} / \mathrm{s}$ result in full detonation. This very clear paper brackets the conditions needed for TATB detonation and shows the usefulness of advanced computer modeling in predicting initiation behavior.

SHK:4, A Comparison of the Shock and Static Compression Curves for Four Solid Explosives. J. J. Dick, J. Energet, Mat. 1, 275-286 (1983),

The shock Hugoniots of PETN, RDX, HMX, and TATB are compared with data from their isothermal static compression curves up to $10 \mathrm{GPa}$. This paper contains a convenient source for the 
isothermal compression curve for TATB up to $9.4 \mathrm{GPa}$. The primary issue of this paper was to determine whether the lower pressure regimes of the shock Hugoniots for these materials corresponds to unreacted material or whether some of the shock strength is due to energy release from chemical reaction. In all four compounds studied, including TATB, the shock Hugondots were shown to be in excellent agreement with the isothermal P.V curves, indicating that the shock response of these explosives in this range is that of the unreacted material.

SHK:5. Stress Induced Radical Generation in TATB. M. Miles, D. Gustaveson, and K. DeVries, J. Mater. Sci. 18, 3243-3248 (1983).

Optical microscopy and electron spin resonance (ESR) were used to examine the extent of compositional degradation of TATB from gamma radiation, mechanical impact, and frictional grinding. The authors observed a strong signal from gamma-irradiated samples identical to that observed by Britt et al. (SPP:8). They also observed a weak ESR signal from such samples that they ascribe to the $\mathrm{NO}_{2}$ radical. TATB'was ground with a mortar and pestle at room temperature and immersed in liquid nitrogen. Strong ESR signals were observed in most samples from both grinding and drop-hammer impact. However, samples with very small particle size did not produce an ESR signal when ground but did produce a signal when subjected to drop-hammer impact. The authors provide some inconclusive results regarding the effects of low temperature on the efficiency of radical generation.

SHK:6. Sub-Ignition Reactions at Molecular Levels in Explosives Subjected to Impact and Underwater Shock. J. Sharma, J. C.Hoffsommer, D. J, Glover, C. S. Coffey, J. W. Forbes, T. P. Liddiard, W. L. Elban, and F. Santiago, Eighth Symposium (International) on Detonation, pp. 725733 (1985).

A number of explosives, including TATB, were exposed to low-intensity shocks (by drop-hammer or Naval Surface Weapons Center aquarium test). The residues were recovered and examined using $x$-ray photoelectron spectroscopy, chemical tonization mass spectroscopy, and thin-layer chromatography. Residue from shocked TATB is shown to have an XPS spectrum in the region of the nitro group $1 \mathrm{~s}$ ionization level that is significantly different from that of unreacted TATB. The authors interpret this finding as reflecting the formation of furazan and furoxan structures resulting from the loss of $\mathrm{H}_{2} \mathrm{O}$ from the molecule. This work is intermediate between that reported in SHK:2 (above) and in SHK:8 (below). The latter includes a complete analysis of TATB by this research team.

SHKi7. Single-Pulse Raman Scattering Studies of Heterogeneous Explosive Materials. W. M. Trott and A. M. Renlund, Appl. Opt, 24, 1520-1525 (1985).

This paper represents one of the very first attempts to employ optical transient spectroscopic techniques to the study of reacting high explosives. The results on TATB are sparse, indicating only the possibility of using stimulated Raman scattering as a probe of detonation reaction chemistry. The experimental success of this technique is realized in SHK:10.

SHK:8. The Physical and Chemical Nature of Sensitization Centers Left from Hot Spots Caused in TA TB by Shock or Impact. J. Sharma, J. W. Forbes, C. S. Coffey, and T. P. Liddiard, 1. Plyys. Chem. 91, 5139-5144 (1987).

Various analytical techniques are used to attempt to identify the detalls of chemical decomposition in sub-initiated TATB samples. This is a fairly significant work in the overall body of literature on TATB shock and thermal decomposition. TATB was shocked in two different ways, either by drop hammer or in the NSWC underwater shock test, which generates a 16- to 18-bar shock for 1 to $2 \mathrm{~s}$. Samples were recovered and examined by SEM and XPS. Two observations are of particular significance. First and most important is a nearly direct observation of the result when "hot spots" are formed within shocked samples. Small holes and residue particles on the order of 0.1 to $5.0 \mu \mathrm{m}$ (depending on the method of impact or shock) were observed in the SEM. The deposits were shown to be soluble in acetone (unlike TATB itself), Second, the XPS spectra collected on the residues clearly showed a loss of $\mathrm{NO}_{2}$ nitrogen $1 \mathrm{~s}$ intensity, indicating that some sort of chemical reartion had taken place within the sample. The new spectrum was not inconsistent with the formation of furoxan and 
furazan products. Although the authors may be overinterpreting their data, which they state as being definitive evidence supporting the formation of these derivatives, there is additional evidence supporting this kind of reactivity in the XPS of the residue that is acetone soluble. The authors indicate that this work, when considered with the results in DTA:7, connects the kind of chemical reactivity observed in shocks to that observed in lower-temperature thermal decomposition.

SHK:9. The Hugoniot and shock sensitivity of a Plastic Bonded Explosive PBX 9502. J. J. Dick, C. A. Forest, J. B. Ramsey, and W. L. Seltz, J. Appl. Phy/s, 63, 4881-4888 (1988).

The authors describe in detall the Hugoniot and run-to-detonation behavior of PBX 9502. Their comments of most interest in this review are those about the anisotropy of the bulk sound speed in the pressed binder/explosive system. Although few data are presented to support the statement, it is argued that the speed of sound obtained by extrapolating the Hugoniot data is anomalously low because of crystallite alignment in the pressed-solid measurement.

SHK:10. Single Pulse Raman Scattering of TATB under Shock Compression. W. M. Trott and A. M. Renlund, J. Phys. Chem. 92, 5921-5925 (1988).

The results of some very difficult single-shot spectroscopic experiments are described. Using a detonator and an inert buffer material as the shock source, the authors recorded single-shot, spontaneous Raman spectra of TATB at pressures up to $7.5 \mathrm{GPa}$. They examined the spectrum in the region of the $\mathrm{NO}_{2}$ deformation mode at $881 \mathrm{~cm}^{-1}$, the $\mathrm{C}-\mathrm{N}_{2} \mathrm{O}_{2}$ symmetric stretching mode at $1146 \mathrm{~cm}^{-1}$, and the symmetric $\mathrm{NO}_{2}$ stretching mode at $1170 \mathrm{~cm}^{-1}$. Failrly good-quality spectra were obtained. The results are compared with those seen in static highwpressure loading in a diamond-anvil cell. The differences between the static and the shock spectra are ascribed to temperature effects associated with the shocked sample. These temperature effects are best manifested by the disruption of the intramolecular hydrogen-bond network and the consequent change in the Raman spectra of the $\mathrm{N}-\mathrm{O}$. bonds. A new Raman line seen at $1120 \mathrm{~cm}^{-1}$ may be due to an intermediate chemical reaction. A possible flaw in the interpretation of the spectrum resides in the questionable vibrational mode assignment of the lines studied. The paper does not cite any of the vibrational studies published to date; however, the assignments of the 881,1140 , and $1176 \mathrm{~cm}^{-1}$ lines do not agree with those of any of the previous analyses. The assignments used in this paper are taken from a personal communication from $\mathrm{B}$. Swanson of Los Alamos.

\subsection{Technical Summary}

This class comprises two different types of study. Several papers investigate the compressibility and mechanical response of TATB as a material. Most of the work tries to identify the chemical nature of TATB samples that have experienced shocks of only modest intensity. The work of Sharma et al. over a period of ten years is particularly notable. However, the ultimate interpretation precisely identifying furoxan and furazan structures as reaction products may be extending the interpretation of the experimental results too far. The XPS results do clearly indicate that the nitro group is by far the more reactive substituent on the TATB ring.

\section{Theory (THE)}

\subsection{Overview}

This section is restricted to studies in which the stability or chemical structure of TATB is directly addressed by various forms of quantum chemical calculations. The first two papers listed are based on 
very low-level, semi-empirical calculations. The later papers use true ab initio calculations, but are limited in their own right.

TATB is a large molecule with an unusual electronic structure. The accuracy that any calculation can achieve is fairly limited, even with the most advanced codes and computer capabillties, None of the calculations done to date can describe detalled properties related to energies of states, potentialenergy curves for reaction coordinates, and other properties directly connected to chemical reactivity. Such values can be reliably calculated only for the simplest molecular systems. These studtes are limited to calculating properties such as bond polarization, overlap populations, and charge distributions within the ring or the substituents. These electronic charge distributions can then be correlated with wh.t are probable reactive sites within the molecule. This is the general approach taken by all of these studtes.

\subsection{Individual Studies}

THE:1. Relation entre la Structure Electronique et la Sensibilite au Choc des Explosifs Secondaire-Nitres-Critere Moleculaire de Sensibilite (Relation between the Electronic Structure and Shock Sensitivity of Secondary Nitro Explosives. Molecular Criteria for Sensitivity.) A. Delpuech and J. Cherville (French, abs. in English), Prop. and Exp. 3, 169-175 (1978).

This is the first of several papers by these atuthors using quantum chemical calculations to explain variations in shock sensitivity of secondary explosives. The main thrust of their theory is the idea that sensitivity is related to the change in polarization of a crucial bond in ta given molecule between its ground and lowest excited electronic state. The authors examine a varlety of nitramines and polynitroaromatics (including TATB) by way of CNDO-S/CI calculations (a fairly low-level computation) of the molecular ground and lowest excited state. 'The correlation between these calculated properties and the shock sensitivity, believed to be initiated by the rupture of the $\mathrm{C}-\mathrm{NO}_{2}$ bond in nitroaromatics and the $\mathrm{N}-\mathrm{NO}_{2}$ bond in nitramines, seems to work at this semi-empirical level. The authors offer no detalled explanation as to why, fundamentally, this correlation should work, beyond several sentences suggesting that the population of an electronic excited state (during a shock) will focus vibrational energy along these critical vibrational modes. The origin of this idea may involve excited state geometry changes. As a direct result of its different electronic structure, the equilibrium geometry of an excited state will, in general, be different from that of a ground state. Rapid excitation into this state from the ground state will initiate motion along the vibrational coordinates that correspond to this difference in geometry. Thus, bonds whose local electronic structure changes the most will receive entanced vibrational excitation and consequently will be more reactive.

THE:2. Relation entre la Structure Electronique et la Sensibilite au Choc des Explosifs Secondaire Nitres. III. Influence de l'environnment cristallin. (Relation between The Electronic Structure and the Shock Sensitivity of Secondary Nitro Explosives. III. Influence of the crystalline environment.) A. Delpuech and J. Cherville (French, abs, in English), Prop. and Exp, 4, 61-65 (1979).

This paper is an extension of the work in THE:1 above. More complicated calculations performed on RDX, HMX, TATB, and other explosives include the effects associated with placing an individual molecule in a crystal lattice. The authors calculate that a crystal lattice produces very little change in charge separation in $\mathrm{C}-\mathrm{NO}_{2}$ or $\mathrm{N}-\mathrm{NO}_{2}$. They conclude that the effect of the lattice is minimal within the scope of their hypothesis. It is highly unlikely that a calculation at the CNDO level can properly incorporate the effects of a lattice, particularly for TATB, whose crystal structure shows such convincing evidence of strong intermolecular interactions.

THE:3. $A b$ initio MODPOT/VRDDO/MERGE Calcuiations on Energetic Compounds. III. Nitroexplosives: Polyaminopolynitrobenzenes (Including DATD, TATB, and Tetryl), P. C Harihan, W. S. Koski, J. J. Kaufman, and R. S. Miller, Int. J. Quant. Chem. 23, 1493-1504 (1983).

This paper reports the details of a number of ab initio calculations performed on DATB, tetryl. TATB, and other explosives. These are much higher-level calculations than those in THE:1 and THE:2. 
The computational technique is one that simpliftes the core electron part of the overall problem to improve computational efficlency. The paper examines gross atomic populations (CAPs) and total overlap populations (TOPs). The GAPs, the authors suggest, represent the net charges on individual atoms in a given molecule. This information is necessary for calculations on multipolar celectrostatic Interactions, which are determined by intermolecular crystal packing forces. The TOP's are related to the overall strength of an individual bond within one molecule. All the aminonitronenzenes are shown to have a much stronger $\mathrm{C}-\mathrm{NH}_{2}$ bond than $\mathrm{C}-\mathrm{NO}_{2}$ bond. The authors express some concern that, despite their interesting qualitative results, the aminonitrobenzenes must be described by a multideterninant wavefunction, and the SCF level of the present calculation may not be adequate to describe the electronic structure of the nitro groups properly. This is a result of the consiclerable electron donating and withdrawing capabilities of the amino and nitro substituents. Simply stated, other resonance structures whose importance was recognized many years ago (STR:1) must be properly considered. The single configuration level does not take these structures into full consideration.

THE:4. Molecular Orbital Calculation of Indices of Impact and Shock Induced Reactivity in Nitroaromatic Molecules. F. J. Owens and P. Polltzer, Shock Ware's in Condensed Matt.1, pp. 857-861 (1985).

This is a preliminary publication of the work presented in THE:5 below. Here the authors propose that (within the group of polynitroaromatics) the total charge on the carbon atom of the weakest bond is correlated with the impact sensitivity of the molecular system. This result is related, but not directly, to the work of Delpuech and Cherville (THE:1,2 above), However, this report does not use any property of electronic excited states of the molecule to justify its conclusions. The most important message is that the insensitive nature of TATB can be explained at a computational level that does not directly include any effects of the crystalline lattice structure or of hydrogen bonding.

THE:5. Computational Analysis of Some Properties Associated with the Nitro Groups in Polynitroaromatic Molecules. F. J. Owens, K. Jayasuriya, L. Abrahmsen, and P. Polizer, Chem, Phys, Lett. 116, 434-438 (1985).

This paper focuses on the polarization of the $\mathrm{C}-\mathrm{NO}_{2}$ bond in a series of nitroaromatic explosives that includes TATB. These SCF calculations were carried out using the GAUSSIAN 80 quantum chemistry program at the STO-3G level. The results indicate that all molecules studied show a significant build-up of positive charge above and below the molecular plane at the $\mathrm{C}-\mathrm{NO}_{2}$ bond. This positive charge is reduced by the addition of amino groups to the ring. The positive charge makes this site on the ring (the $\mathrm{C}-\mathrm{NO}_{2}$ bond) susceptible to nucleophilic attack. This paper also examines $\mathrm{C}-\mathrm{NO}_{2}$ bond polarity. A very clear correlation is found between a parameter related to this bond polarity and results of drop-hammer and shock sensitivity tests. The paper indicates that the stability of TATB can be explained without any reference at all to hydrogen bonding. The authors discuss a possible explanation for curiosities in the XPS (DTA:7) of several of the molecules.

\subsection{Technical Summary}

All of these papers focus on the $\mathrm{C}-\mathrm{NO}_{2}$ bonds and their reactivity within a series of aminosubstituted compounds. The earlier work by Delpuech and Cherville was based on an assumption that shock sensitivity is related to characteristics of electronic excited states. Later work does not consider electronic excited states, and in general there is not a lot of support for the idea that the electronic structure of excited states has much to do with shock reactivity. However, Delpuech and Cherville's work does emphasize the correlation between reactivity and properties associated with bond polarity. The later calculations continue this notion and draw some successful relationships between reactivity and $\mathrm{C}-\mathrm{N}$ charge distributions. It is important to note, however, that all of these papers have assumed that the rupture of the $\mathrm{C}-\mathrm{NO}_{2}$ bond is a key step in controlling the reactivity in the entire class of nitroaromatics. No other reaction mechanisms are considered.

Other reaction mechanisms have been proposed by experimentalists; specifically, ring depolymerization (D'TA:6) and dehydration, and subsequent outer ring closure: These concerted 
pathways, invalving bonds linking many nuclel, cannot be evaluated at the present computational level. The quantum chemistry methods presc ated here do not calculate energles of different reaction pathways along various reactlon coordinates, which would constitute a cleaner theoretical treatment of the problem. In a large molecule such as TATB this ts not possible. Instend, these studies calculate a static electronic structure with a known crystallographle geometry and then relate the electronic structural properties to observed renctivity trends. These treatments should not be taken as directly supporting $\mathrm{C}-\mathrm{NO}_{2}$ bond cleavage as the inttial reaction pathway of lowest energy for TATB. However, the calculations do support substituent reactivity (dehydration or direct $\mathrm{NO}_{2}$ loss) as taking precedence over concturted ring break- $u_{p}$,

\section{Overall Summary}

Most of the work reported in this review was accomplished over the past fifteen yrars. The years from 1981 to 1985 were particularly fruttful, especially with regard to the use of newer llagnostic techniques such as mass spectroscopy and photoelectron spectroscopy. It seems that today there is less work going on throughout the Department of Defense, the Department of Energy, and academia than there was ten years ago. It is hoped that the reason for the decrease in activity is due to the simple fact that most of the questions regarding TATB's thermochemtstry and its relationship to shock sensitivity have been answered. This may be partly true, but not entirely. In this last section, we address the most important questions that motivated the work presented in this review.

The questions are these: (1) What is the rate-limiting reaction step in the thermal decomposition of TATB? (2) How are the chemical reaction kinetics of this step related to the shock sensitivity of TATB and its common formulations? (3) How is this "cruclal" reaction step, tf there is only one, related to molecular and crystalline structure? (4) Is this system unique?

We first wish to know how TATB decomposes. We would expect experimental work to identify the simple facts of 'TATB's reaction steps in some general order, with a rough idea of the competitive rates. The next two issues involve generating some understanding from these facts, both utilttarlan and theoretical, respectively. Question (2) asks how microscopic reaction properties such as shock intiation characteristics and critical lemperature are related to the complex bulk properties of the material. Without an understanding of how this chemistry scales up to bulk behavior, the freestanding facts answering question (2) are of only academic interest. Question (3) addresses the fundamental issue of whether the simple facts identifying a known reaction mechanism for a particular system can be explained. Is there a theory that can describe the relationship between electronic structure and reactivity in this complicated organic system containing a variety of heteroatoms and unusual crystalline properties? Can these properties be calculated? Question (4) asks simply "Can we do better?" Is there a possibility of inventing a new. molecular system that has the desirable stability of an IHE but with increased energy and detonatlon velocity? We address each of these questions below.

Question (1). The two frequently suggested initial steps are either direct $\mathrm{NO}_{2}$ loss from the ring or concerted elimination of $\mathrm{H}_{2} \mathrm{O}$ from adjacent nitro and amino groups to form furazan and furoxan-like structures. A third mechanism, concerted ring fisstire, is also a remote possibility.

Direct $\mathrm{NO}_{2}$ loss seems like a simple explanation. It is a first-order endothermic step that would be consistent with some of the multi-step decomposition models. However, there is little direct experimental evidence indicating that this is the appropriate mechanism. Nitrogen dioxide is not seen in low-temperature thermolysis mass spectroscopic studies, and a mechanism such as this is not consistent with residue analysis by infrared spectroscopy. Some photoclectron spectroscopy on partially reacted residues supports this as a first step; however, most of the XPS results could be consistent with almost any mechandsm invoking reactivity at the $\mathrm{NO}_{2}$ site. It is important to note that the theoretical work that correlates calculatec $\mathrm{C}-\mathrm{NO}_{2}$ bond polarization with shock sensitivity does not necessarily mean that $\mathrm{C}-\mathrm{N}$ bond fission is the reaction step. The XPS work shows that the $\mathrm{NO}_{2}$ nitrogen changes oxidation state in an early reaction step. This suggests that reactivity at this site may still be important without implying complete bond rupture and that trends related to nearby electronic structure may still be valid. 
A second mechanism suggested postulates a concerted dehydration ronction that releases water and forms a bond between adjacent amino and nitro substituents. Catalano et al. (TTX:10,11) frled very hatd to find evidence for this mechandsm. None was found. The evidence supporting arguments for this reaction has been put forth primarily by Sharma et al. Most of this work. is based on photoelectron spectra of reaction residues. Photoelectron spectroscopy is not the analytical method of cholce for identifying chemical reaction products. The expertmental results in most of this work are inclusive.

Direct ring breakup is supported by one experiment. This is the work presented in DTA:6, where $\mathrm{C}_{3} \mathrm{~N}_{3}$ and $\mathrm{C}_{2} \mathrm{~N}_{2}$ fragments appear in a mass spectrometer. Other mass spectroscoplc work is inconsistent with this result. Although a likely mechanism for HMX and RDX, ring fragmentation does not find much support as an inttial step in the case of TATB. There expertments should be repeated by another laboratory to enhance their credibillty,

The iritial decomposition reaction of TATB in the condensed phase is probably not the elimination of $\mathrm{H}_{2} \mathrm{O}$. There is definitely not enough evidence to draw such a conclusion. The first step does, however, involve reactivity at the $\mathrm{NO}_{2}$ substituent, and certainly produces a condensed-phase intermediate that is only a partially reacted form of TATB. Several kinetic studies suggest that this reaction could be autocatalytic.

Our review of the evidence suggests that the first step is direct loss of $\mathrm{NO}_{2}$. However, low-level stimull (weak shock, mild heating, etc.) do not disrupt the crystalline lattlce. Instead, the $\mathrm{NO}_{2}$ attacks a neighboring TATB molecule and somehow enhances its decompositton reactivity, it is the network lattlice of TATB that keeps it solid and intact at elevated temperatures. Dissociation and subsequent attack are slow as long as TATB remains solid and tits lattice is preserved. At higher levels of local heat release, the lattice structure is lost, $\mathrm{NO}_{2}$ becomes more mobile, and TATB can react in an exothermic fashion similar to any other CHNO explosive.

Question (2). This can be asked of any explosive. That is, what is the relationship between shock sensitivity and thermal chemical reactivity? Bulk TATB has many properties that are only very indirectly related to the details of its molecular structure. The study of how mechanlal shock energy winds up as heat driving a chemical reaction is a field all its own. In the case of TATB, however, evidence from reclaimed samples suggests that the same rasidues present in weakly shocked samples are also there in thermolysis samples. There is some eviuence that level of confinement can play a role in what is observed as "early products." It is not clear whether experiments at different levels of confinement measure different stages of the same uniform reaction pathway oi whether maintaining products in contact with reactants produces new autocatalytic decomposition pathways that would otherwise not occur.

Each individual molecule certainly undergoes numerous reaction steps before it is converted to final products. In what order these steps occur, and how they interact in a highly confined, high-density environment, is not known for any energetic materiai. TATB is no exception. Most of the results establish that TATB decomposes in the solid phase even at elevated temperature. It is likely, then, that solid-state thermal reactions are similar to those in a shocked system, and that an understanding of thermal response is directly related to an understanding of shock-induced reactivity.

Question (3). Much of TATB's unusual stability has been ascribed to several aspects of its crystal structure. One of these is the network of hydrogen bonding, both within an individual molecule and within a molecular plane, that produces a graphitic planar structure in the overall laltice. In addition, the crystal structure shows a very distorted molecular ring, indicating that overall aromaticity in the carbon frame is reduced and that the carbon sites have an unusual charge distribution.

Explaining TAT'B's stability by Invoking the water-abstraction reaction mechanism appears to be well set up for what is known about the TATB structure. It is easy to see how the hydrogen atoms of a neighboring amino group could remove a nitro oxygen followed by closure of a tive-membered ring. Furthermore, the product of such a reaction is a stable, known molecule. Invoking a reaction pathway that is slowed by a step that produces a relatively unreactive intermediate (that is, relative to free radicals) provides a good argument for the stabillty of TATB.

Direct $\mathrm{NO}_{2}$ loss, as a general first step in decomposition, is also supported by known properties of the structure. The distorted ring and the presence of three amino groups that donate electron density 
back Into the ring can add stabllizing, londc bonding features to the TATB $\mathrm{C}-\mathrm{NO}_{2}$ bond, at least with respect to other members of a homologous series of substituted nitroaromatics.

It can also be suggested that the graphittic crystal structure allows molecular planes to slide under shock loading, preventing the formation of high-temperature ignition centers ("hot spots"). In addition, this structure, which results in a bulk thermal conductivity twice that of most other HEs, can more effectively move hent away from such centers and prevent these sites from runaway exothermic reaction.

There are plenty of explanations for TATB's pecullarttles. They are based on the properties of its structure not only as a free molecule but as a component of a spectal type of lattice array. Unfortunately, these explanations can be made to correlate with many different chemical and structural reasons accounting for TA'TB's stability, It is not clear whether one property dominates the explanation or whether TATB is special because of the sum of a number of contributing effects.

Question (4). TATB is certainly different from other energetic materials, Whether it is unique is difficult to answer. If Its stability comes from single-molecule properties alone, such as intramolecular hydrogen bonding and amino substitution on an aromatic ring that stabilizes nitro group reactivity, then there are probably other molecules, perhaps with heteromatoms in the ring, that exhibit untusual stability, It is possible to identify candidate molecules and to synthesize them.

However, if sensitivity properties are more closely connected to the intermolecular makeup of the lattice, including hydrogen bonding and sheet-like lattlce structure, the situation is different. Since it is currently impossible to predict a resulting crystal structure simply by knowing a molecular structure, It will be very difficult to generate target systems with properties, such as a high melting point and high thermal conductivity, that originate from a graphitic layered lattice. If this is so, TATB may very well be the optimum pure material that combines high energy with exceptional shock and thermal stability.

In this report we have reviewed several types 'of studies, all of which were designed to advance an understanding of the thermal response of a very interesting energetic material, TATB. Our main message is that no final conclusions can yet be drawn regarding the "reasons" for TATB's unique stability. However, the potential roles of at least some molecular and lattice factors have been. identified. We hope that this review can serve as a guide to future studius of this system and will prevent the premature promulgation of interpretations based more on opinion than on experimental evidence.

\section{Acknowledgment}

The authors would like to express their thanks to Dr. J. Richardson and Mr. P. Van Dyke for their efforts in editing and assembling this manuscript.

\section{Bibliography}

Dobratz, B. M., LLNL Explosives Handbook: Properties of Chemical Explosives and Explosive Simulants, Lawrence Livermore National Laboratory, Livermore, CA, UCRL-52997 (March 16, 1981).

Dobratz, B. M., M. Finger, L. G. Green, J. R. Humphrey, R. R. McGuire, and H. F. Rizzo, Selected Sensitivity Tests of TATB and TATB Formulations and their Evaluation, Lawrence Livermore National Laboratory, Livermore, CA, UCID-18026 (1979),

Gibbs, T. R. and A. Popolato, LASL Explosives Properties Data (University of Callfornia Press, Berkeley, CA, 1980).

Mader, C. L., J. N. Johnsón, and S. L. Crane, Los Alamos Explosives Performsnce Data (Los Alamos National Laboratory, Los Alamos, NM, 1982). 
